\title{
Chemotherapy-Associated Liver Injuries: Unmet Needs and New Insights for Surgical Oncologists
}

\author{
Luca Vigano, $\mathrm{MD}, \mathrm{PhD}^{1,3}$ (]) Martina Sollini, $\mathrm{MD}, \mathrm{PhD}^{2,3}$, Francesca Ieva, $\mathrm{PhD}^{4,5}$, Francesco Fiz, $\mathrm{MD}^{2}$, and \\ Guido Torzilli, $\mathbf{M D}, \mathbf{P h D}^{1,3}$ \\ ${ }^{1}$ Division of Hepatobiliary and General Surgery, Department of Surgery, IRCCS Humanitas Research Hospital, Rozzano, \\ Milan, Italy; ${ }^{2}$ Department of Nuclear Medicine, IRCCS Humanitas Research Hospital, Rozzano, Milan, Italy; ${ }^{3}$ Department \\ of Biomedical Sciences, Humanitas University, Pieve Emanuele, Milan, Italy; ${ }^{4}$ MOX laboratory, Department of \\ Mathematics, Politecnico di Milano, Milan, Italy; ${ }^{5}$ CADS - Center for Analysis, Decisions and Society, Human \\ Technopole, Milan, Italy
}

Chemotherapy-associated liver injuries (CALI) were the focus of several research studies some years ago when they were associated with modern treatments for colorectal metastases and operative outcomes of liver resection. ${ }^{1,2}$ An intensive, multidisciplinary commitment was designed to elucidate their pathogenesis, risk factors, clinical impact, diagnosis, and prevention. Then, long before reaching a full understanding, the interest in CALI rapidly decreased. The definition itself of CALI remains uncertain. While sinusoidal dilatation and nodular regenerative hyperplasia (NRH) are unequivocally associated with oxaliplatin-based chemotherapy regimens, this is not the case for steatohepatitis. ${ }^{1,3}$ For the latter, a major, if not exclusive, impact of metabolic disorders rather than of irinotecan has been outlined. $^{4,5}$

CALI are still part of our clinical practice. Moderate-tosevere sinusoidal dilatation is evident in more than onethird of patients undergoing liver surgery after oxaliplatinbased chemotherapy. Steatohepatitis occurs in only $10 \%$ of patients undergoing irinotecan-based chemotherapy but gains major epidemiological interest when its metabolic risk factors are considered (at least one-third of the entire U.S. population is at risk because of metabolic syndrome). ${ }^{5}$

(C) Society of Surgical Oncology 2021

First Received: 2 July 2020

Accepted: 14 April 2021;

Published Online: 30 April 2021

L. Vigano, $\mathrm{MD}, \mathrm{PhD}$

e-mail: luca.vigano@hunimed.eu

\section{CALI DIAGNOSIS: A RIDDLE TO SOLVE}

Standard imaging modalities can demonstrate steatosis, but not steatohepatitis. Liver parenchyma heterogeneity at magnetic resonance imaging (MRI) has been associated with sinusoidal dilatation, as well as splenomegaly and thrombocytopenia, but their evaluation remains uncodified. ${ }^{6,7}$ Some authors reported a correlation between liver atrophy after chemotherapy and CALI, but data are not univocal. ${ }^{8,9}$ The roles of both ultrasound-based and magnetic resonance-based elastography have been investigated, but neither modality can reliably discriminate steatohepatitis from simple steatosis. ${ }^{10}$ Even liver biopsy failed to diagnose CALI other than steatosis because of heterogeneous distribution of injuries and limited tissue sample. ${ }^{11}$ So far, we have only been able to approximately predict CALI through clinical data, i.e., chemotherapy regimen and number of administered cycles, and some liver function tests, including APRI score, ICG test, and LiMAx test.

Surgeons need a more robust assessment of CALI. While liver function tests are markedly impaired during chemotherapy and in the early postchemotherapy period, they tend to normalize within 4-6 weeks after the end of treatment. Normalization may occur later when prolonged chemotherapy is administered, but CALI last for a long time: sinusoidal dilatation and $\mathrm{NRH}$ require at least 9 months to disappear, while steatosis and steatohepatitis persist even later on. ${ }^{12}$ Liver function tests are completely normal in up to one-third of patients with CALI, leading to underestimation of postoperative risk. 
Clinicians have further reasons to pursue CALI diagnosis. This is mandatory to assess the effectiveness of any measure to reduce or prevent liver injuries. Second, diagnosis is needed in case of recurrent disease when patients require new chemotherapy lines and repeated surgery. CALI are crucial to predict tolerance to treatment and operative risks. Finally, the identification of CALI is needed as long as they have some oncological impact. Even if not associated with prognosis, some studies highlighted an inverse relationship between CALI and response to chemotherapy (tumor regression is less evident in patients with severe injuries). ${ }^{3,13}$

\section{NEW OUTLOOKS: FUNCTIONAL IMAGING AND TEXTURE ANALYSIS}

Hepatobiliary scintigraphy (HBS) with ${ }^{99 \mathrm{~m}}$ Tc-labeled iminodiacetate derivatives could open new perspectives. In staged procedures, HBS demonstrated accurate evaluation of liver function, outperforming volumetric assessments and liver function tests. ${ }^{14,15}$ The hypothesis is appealing: if HBS is able to identify heterogeneous liver function it also could accurately depict the presence, distribution, and functional impact of CALI. To date, only one paper explored this topic, and the results were not completely satisfactory. ${ }^{16}$ HBS unveiled a reduced liver function of the future liver remnant (FLR) in patients with steatosis $\geq 30 \%$ and those with NAS (Nonalcoholic fatty liver disease Activity Score) $>1$, but not in those with sinusoidal dilatation. HBS measures hepatocellular function and could be able to predict only hepatocyte-related diseases, but its true potentialities have not been fully explored. In the Truant et al. paper less than 5\% of patients had grade 2-3 sinusoidal dilatation precluding any conclusive evaluation, and NRH, a highly relevant CALI, was not even taken into account. ${ }^{7}$ A cohort of patients with an adequate prevalence of CALI could achieve positive data.

A further contribution could derive from digital images analysis and processing. They have so far persistently been proven to include invisible-to-the-human-eye texture characteristics that can be correlated with pathology data and outcomes. ${ }^{17}$ Some evidence has been advanced for radiomic analyses in colorectal metastases, but none concerned CALI. ${ }^{18}$ Texture information has revealed itself to be a proxy of shape, heterogeneity, and granularity of biological tissue and, consequently, potential predictor of liver parenchyma alterations. However, the close-source nature of radiomics, alongside unharmonized acquisition settings, discordant reconstruction parameters, lack of interpretability, and methodological biases result in the standard radiomic approach being scarcely flexible and robust. The direct analysis of raw data could enable clearer and more unspoiled findings. Preliminary results from our investigation show promising evidence of such correlation. Raw baseline computer tomography images were reconstructed and grey levels adjusted according to Hounsfield units scale, before extraction of volumes of interest (VOIs). VOIs consisted of standardized samples of healthy liver tissue, potentially affected by CALI. Such VOIs were unfolded to obtain a one-dimensional representation of the portion and three features entailing a homogeneity score in tissue texture, with an increasing level of detail, were explored. Two patterns per analysis can be appreciated (Fig. 1). Interestingly, the two patterns exactly correspond to patients with and without severe CALI.

\section{COMPASS FOR SURGICAL ONCOLOGISTS}

CALI have a proven negative impact on the operative outcome, increasing both major complication and liver failure rates, even in presence of apparently normal liver function. ${ }^{19}$ Thus, surgeons must adopt a caution-driven strategy. We propose a five-step approach not to overlook any available tool and warning (Fig. 2). First, CALI should be prevented by scheduling short preoperative chemotherapy, i.e., no more than six cycles, and their negative impact should be limited by respecting an adequate chemotherapysurgery interval, i.e., at least 4 weeks. ${ }^{20,21}$ Do not forget that selected patients may undergo surgery without perioperative chemotherapy, as also suggested by the international guidelines. ${ }^{22}$ No therapy for CALI is available, but some reversibility of steatosis has been obtained in living-donor liver transplantation candidates through health regimen and correction of metabolic disorders, even after a short treatment. ${ }^{23}$ Could prehabilitation before liver resection reduce liver injuries and their negative impact on outcome?

Second, when surgery is scheduled, risk factors for CALI should be kept in mind, namely chemotherapy regimen and associated metabolic disorders. Preoperative imaging modalities should be checked for hallmarks of liver injuries, including liver atrophy after chemotherapy, parenchyma heterogeneity at MRI, and splenomegaly. ${ }^{6,8,9}$ APRI score should be computed and additional liver function tests (e.g., ICG or LiMAx test) should be performed according to local availability. All these data taken together do not provide a conclusive diagnosis but define a risk profile for each patient.

Third, as long as CALI compromise liver function, the best way to limit their impact is parenchyma-sparing surgery. Minor hepatectomies are no more synonymous with simple resections and can be considered for complex disease presentations. ${ }^{24,25}$ It is obvious that the higher the proportion of major hepatectomies the higher the severe 
Digital medical image

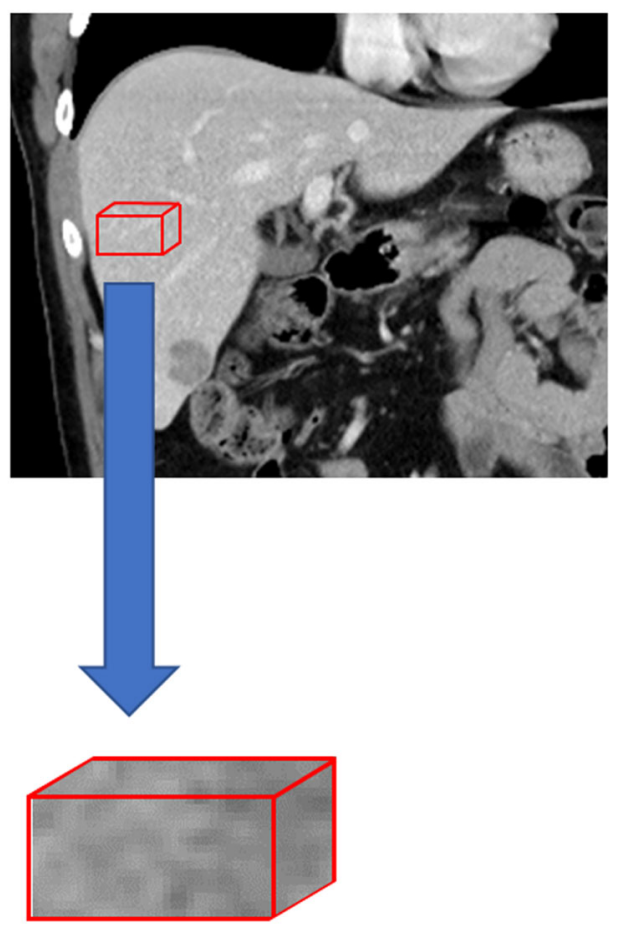

8 VOIs from 8 patients
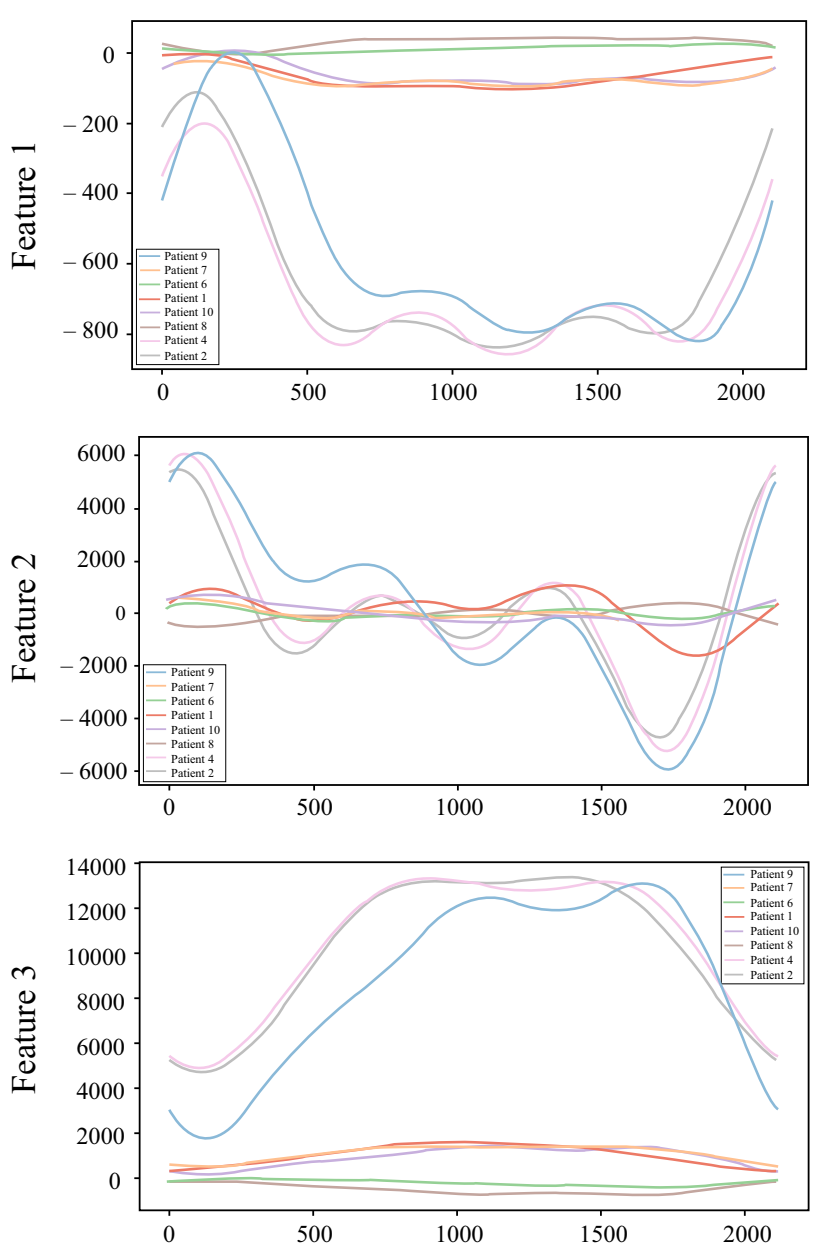

representation of the portion; feature \#2: volumes were then derived, applying a Sobel filter to the three directions, and unfolded to create a one-dimensional metrics of neighbor grey level first-order variability; feature \#3: Laplacian of Gaussian filter was performed on volumes such that, after unfolding, one-dimensional second-order variability measure among neighborhoods of grey level was built for each patient's study. Patients with CALI (\#2, 4, and 9) and without CALI $(\# 1,6,7,8$, and 10) have a different pattern of all features

threshold to $30 \% .^{7,16,26-29}$ Even if these data did not correspond to an increased mortality rate ( $0 \%$ in most series), they suggest the need for an extension of the indications to preoperative portal vein occlusion. Robust evidence is still to provide. Ferrero et al. reported no liver failure in patients with FLR $>35 \% .{ }^{29} \mathrm{We}$ adopt a higher cutoff value, i.e., $40 \%$, in agreement with the Eastern centers. ${ }^{30}$ Additional features could be considered, such as the postchemotherapy liver atrophy, and the remnant growth rate after portal vein occlusion, but they are not yet part of current clinical practice. ${ }^{8,31}$ A more sophisticated approach could modulate the cutoff value of FLR volume according to the results of liver function tests. Even if appealing, this approach has been poorly explored. Takamoto et al. proposed an FLR volume $>60 \%$ in patients with ICG test $\geq 10 .^{30}$ Such a high threshold could be difficult to conciliate with modern $25 \%$, and is between 10 and $15 \%$ when they rise the 

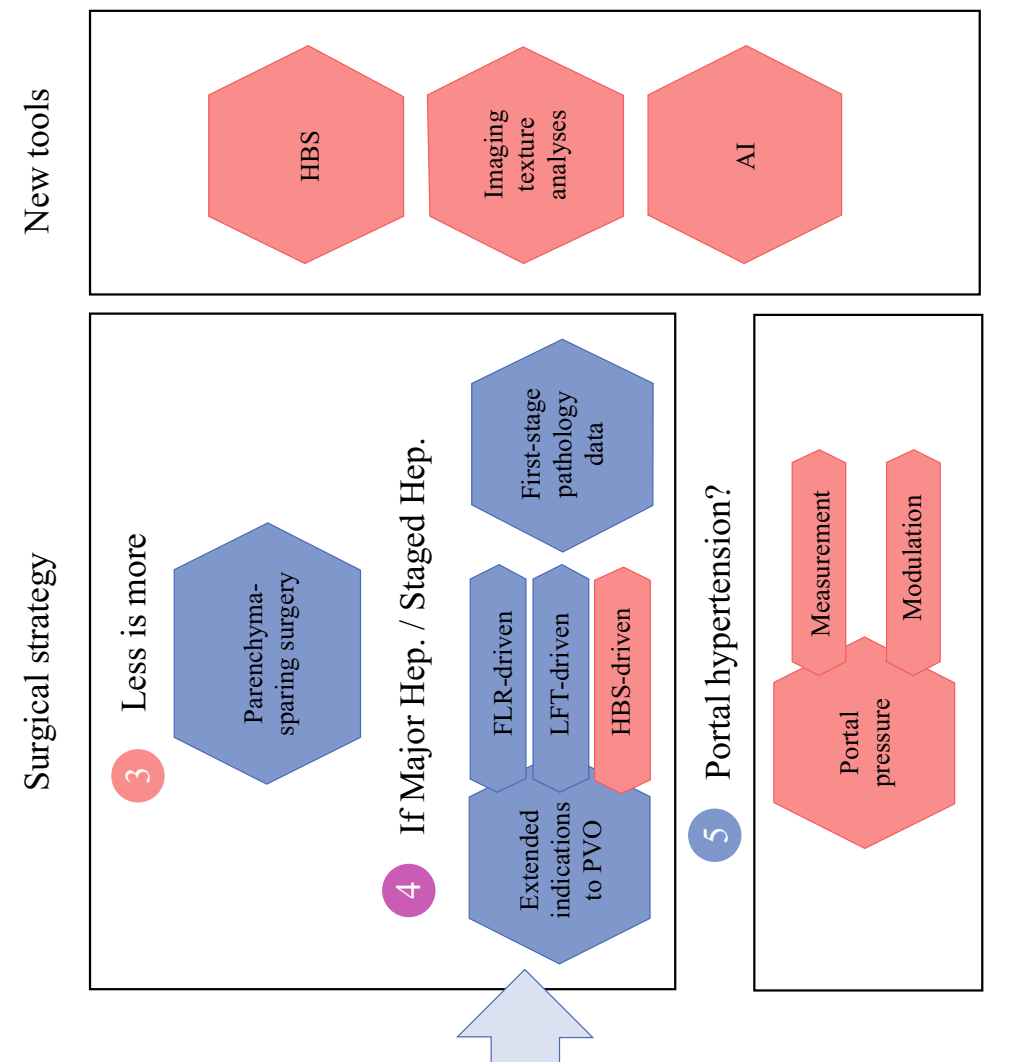

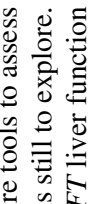

至点这

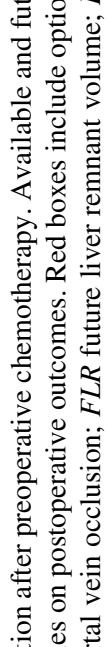

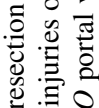

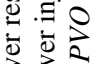

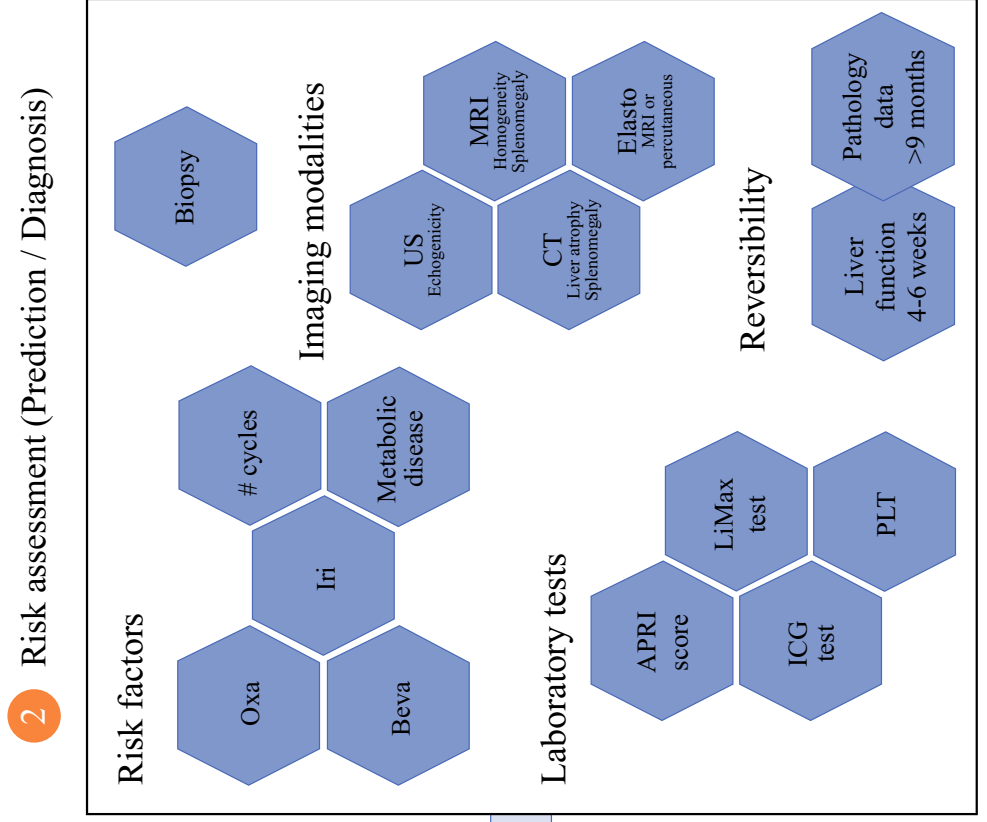

$\stackrel{\Xi}{*}$

पे

吾害造

$\frac{0}{0} \stackrel{0}{0}$

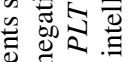

苋完

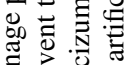

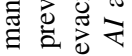

0 응

产.

莺芯昰

50

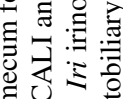

可记

《

$\checkmark$ 这范

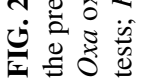

:
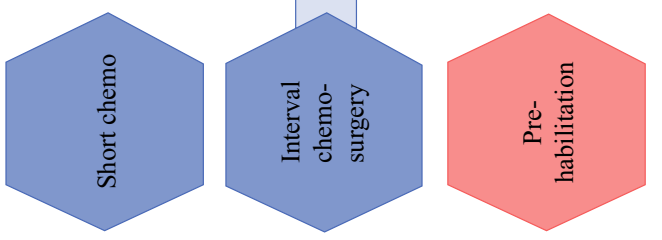


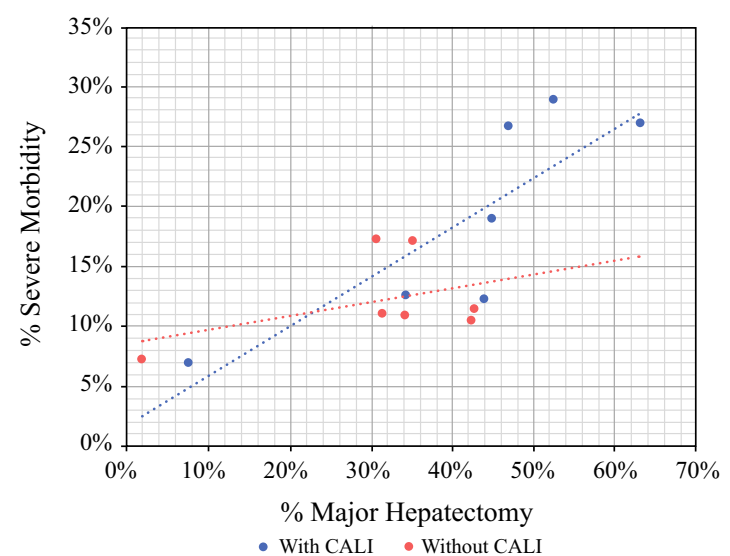

FIG. 3 Correlation between the proportion of major hepatectomies and the severe morbidity (a) and postoperative liver failure rate (b) in the published series. Data were extracted from the following studies: Nakano et al., Ann Surg. 2008;247:118-24; Soubrane et al., Ann Surg. 2010;251:454-60; Wicherts et al., Ann Surg Oncol. 2011;18:659-69;

aggressive surgical indications, especially when staged procedures are scheduled. Obviously, if a staged hepatectomy is planned, the evaluation of CALI on the specimen of the first hepatectomy should drive decisions concerning the second-stage procedure. The association between the CALI, FLR value and outcomes in patients undergoing staged procedures remains unexplored.

Fifth, sinusoidal injuries, namely severe NRH, may lead to portal hypertension. ${ }^{7}$ If suspected, preoperative assessment of hepatic venous pressure gradient could be considered, as well as intraoperative measurement of portal pressure and portal flow modulation.

\section{TOWARD A PRECISION SURGERY}

So far, surgeons are aware that liver function, histology, and volume express different nuances that have to be separately weighted, and then combined together and with the other patients' characteristics to obtain the whole picture, to accurately predict outcomes, and to design the most adequate approach. In this sense, HBS and texture analyses could consistently be two major breakthroughs. HBS unveils any functional heterogeneity of liver parenchyma and provides a functional volumetry of the FLR that could further refine indications to preoperative portal vein occlusion. On the other hand, image mining and analysis enables the performance of a "virtual biopsy" of the liver that could lead to a reliable CALI diagnosis. Furthermore, radiomic features could be per se biomarkers of liver function and predictors of postoperative outcome, as anticipated by some preliminary studies. ${ }^{32,33}$ The next step forward, worth mentioning, is the exploration of artificial intelligence protocols. They could combine clinical, laboratory, volumetric, and imaging data into a single

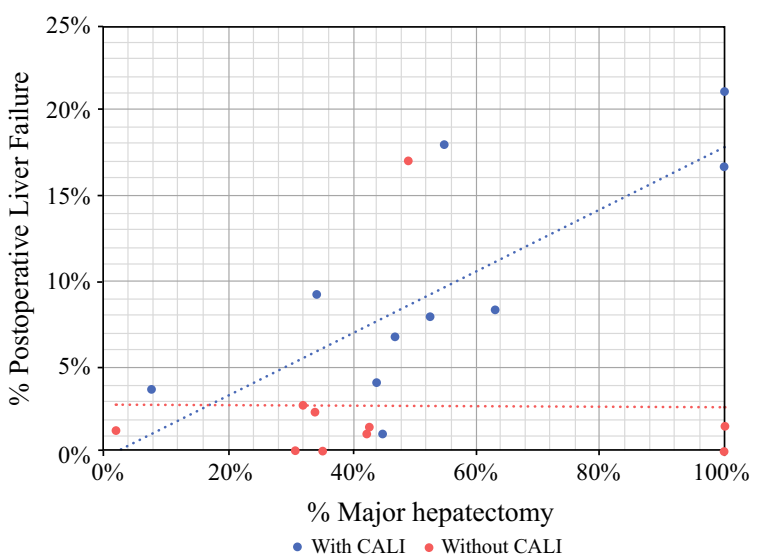

Viganò et al., Ann Surg Oncol. 2015;22:4149-57; Viganò et al., J Hepatol. 2017;67:84-91; Zhao et al., Br J Surg. 2017;104:990-1002; Shindoh et al., Ann Surg Oncol. 2019;26:4100-7; Truant et al., Ann Surg Oncol. 2021;28:1959-69; Viganò et al., submitted data.

predictive model that can provide a patient-tailored surgical strategy. These are consistently the basis for a precision medicine approach. Premises are enough to fuel a renewed interest in CALI research.

ACKNOWLEDGMENT The authors thank Lara Cavinato for her contribution to images' analysis and Patricia Taylor for editing a draft of the manuscript.

\section{REFERENCES}

1. Rubbia-Brandt L, Audard V, Sartoretti P, et al. Severe hepatic sinusoidal obstruction associated with oxaliplatin-based chemotherapy in patients with metastatic colorectal cancer. Ann Oncol. 2004;15(3):460-6. https://doi.org/10.1093/annonc/mdh 095.

2. Vauthey JN, Pawlik TM, Ribero D, et al. Chemotherapy regimen predicts steatohepatitis and an increase in 90-day mortality after surgery for hepatic colorectal metastases. J Clin Oncol. 2006;24(13):2065-72. https://doi.org/10.1200/JCO.2005.05. 3074.

3. Viganò L, Capussotti L, De Rosa G, De Saussure WO, Mentha G, Rubbia-Brandt L. Liver resection for colorectal metastases after chemotherapy: impact of chemotherapy-related liver injuries, pathological tumor response, and micrometastases on long-term survival. Ann Surg. 2013;258(5):731-42. https://doi.org/10.1097/ SLA.0b013e3182a6183e.

4. Ryan P, Nanji S, Pollett A, et al. Chemotherapy-induced liver injury in metastatic colorectal cancer: semiquantitative histologic analysis of 334 resected liver specimens shows that vascular injury but not steatohepatitis is associated with preoperative chemotherapy. Am J Surg Pathol. 2010;34(6):784-91. https://doi. org/10.1097/PAS.0b013e3181dc242c.

5. Sheka AC, Adeyi O, Thompson J, Hameed B, Crawford PA, Ikramuddin S. Nonalcoholic steatohepatitis: a review. JAMA. 2020;323(12):1175-83. https://doi.org/10.1001/jama.2020.2298.

6. Zhang Y, Yan Y, Song B. Noninvasive imaging diagnosis of sinusoidal obstruction syndrome: a pictorial review. Insights Imaging. 2019;10(1):110. https://doi.org/10.1186/s13244-019-07 91-x. 
7. Viganò L, Rubbia-Brandt L, De Rosa G, et al. Nodular regenerative hyperplasia in patients undergoing liver resection for colorectal metastases after chemotherapy: risk factors, preoperative assessment and clinical impact. Ann Surg Oncol. 2015;22(13):4149-57. https://doi.org/10.1245/s10434-015-45330.

8. Shindoh J, Kobayashi Y, Kinowaki K, et al. Dynamic changes in normal liver parenchymal volume during chemotherapy for colorectal cancer: liver atrophy as an alternate marker of chemotherapy-associated liver injury. Ann Surg Oncol. 2019;26(12):4100-7. https://doi.org/10.1245/s10434-019-07740$x$.

9. Tani K, Shindoh J, Takamoto T, et al. Kinetic changes in liver parenchyma after preoperative chemotherapy for patients with colorectal liver metastases. $J$ Gastrointest Surg. 2017;21(5):813-21. https://doi.org/10.1007/s11605-017-3359-4.

10. Castera L, Friedrich-Rust M, Loomba R. Noninvasive assessment of liver disease in patients with nonalcoholic fatty liver disease. Gastroenterology. 2019;156(5):1264-81.e4. https://doi.org/10.10 53/j.gastro.2018.12.036.

11. Viganò L, Ravarino N, Ferrero A, Motta M, Torchio B, Capussotti L. Prospective evaluation of accuracy of liver biopsy findings in the identification of chemotherapy-associated liver injuries. Arch Surg. 2012;147(12):1085-91. https://doi.org/10.10 01/archsurg.2012.1867.

12. Vigano L, De Rosa G, Toso C, et al. Reversibility of chemotherapy-related liver injury. J Hepatol. 2017;67(1):84-91. https://doi.org/10.1016/j.jhep.2017.02.031.

13. Zhao J, Sawo P, Rensen SS, et al. Impact of chemotherapy-associated liver injury on tumour regression grade and survival in patients with colorectal liver metastases. HPB (Oxford). 2018;20(2):147-54. https://doi.org/10.1016/j.hpb.2017.08.030.

14. Olthof PB, Tomassini F, Huespe PE, et al. Hepatobiliary scintigraphy to evaluate liver function in associating liver partition and portal vein ligation for staged hepatectomy: liver volume overestimates liver function. Surgery. 2017;162(4):775-83. http s://doi.org/10.1016/j.surg.2017.05.022.

15. Serenari M, Collaud C, Alvarez FA, et al. Interstage assessment of remnant liver function in ALPPS using hepatobiliary scintigraphy: prediction of posthepatectomy liver failure and introduction of the HIBA Index. Ann Surg. 2018;267(6):1141-7. https://doi.org/10.1097/SLA.0000000000002150.

16. Truant S, Baillet C, Gnemmi V, et al. The impact of modern chemotherapy and chemotherapy-associated liver injuries (CALI) on liver function-value of $99 \mathrm{mTc}$-labelled-mebrofenin SPECThepatobiliary scintigraphy. Ann Surg Oncol. 2021;28(4):1959-69. https://doi.org/10.1245/s10434-020-08988-4.

17. Sollini M, Antunovic L, Chiti A, Kirienko M. Towards clinical application of image mining: a systematic review on artificial intelligence and radiomics. Eur J Nucl Med Mol Imaging. 2019;46(13):2656-72. https://doi.org/10.1007/s00259-019-04372 $-\mathrm{x}$.

18. Fiz F, Viganò L, Gennaro N, et al. Radiomics of liver metastases: a systematic review. Cancers. 2020;12(10):2881. https://doi.org/ $10.3390 /$ cancers 12102881

19. Zhao J, van Mierlo KMC, Gómez-Ramírez J, et al. Systematic review of the influence of chemotherapy-associated liver injury on outcome after partial hepatectomy for colorectal liver metastases. Br J Surg. 2017;104(8):990-1002. https://doi.org/10. 1002/bjs. 10572

20. Karoui M, Penna C, Amin-Hashem M, et al. Influence of preoperative chemotherapy on the risk of major hepatectomy for colorectal liver metastases. Ann Surg. 2006;243(1):1-7. https://d oi.org/10.1097/01.sla.0000193603.26265.c3.

21. Welsh FK, Tilney HS, Tekkis PP, et al. Safe liver resection following chemotherapy for colorectal metastases is a matter of timing. Br J Cancer. 2007;96(7):1037-42. https://doi.org/10.103 8/sj.bjc.6603670.

22. Van Cutsem E, Cervantes A, Adam R, et al. ESMO consensus guidelines for the management of patients with metastatic colorectal cancer. Ann Oncol. 2016;27:1386-422.

23. Nakamuta M, Morizono S, Soejima Y, et al. Short-term intensive treatment for donors with hepatic steatosis in living-donor liver transplantation. Transplantation. 2005;80(5):608-12. https://doi. org/10.1097/01.tp.0000166009.77444.f3.

24. Viganò L, Torzilli G, Troisi R, et al. Minor hepatectomies: focusing a blurred picture: analysis of the outcome of 4471 open resections in patients without cirrhosis. Ann Surg. 2019;270(5):842-51. https://doi.org/10.1097/SLA. 0000000000003493.

25. Torzilli G, Viganò L, Gatti A, et al. Twelve-year experience of "radical but conservative" liver surgery for colorectal metastases: impact on surgical practice and oncologic efficacy. $H P B$. 2017;19(9):775-84. https://doi.org/10.1016/j.hpb.2017.05.006.

26. Soubrane O, Brouquet A, Zalinski S, et al. Predicting high grade lesions of sinusoidal obstruction syndrome related to oxaliplatinbased chemotherapy for colorectal liver metastases: correlation with post-hepatectomy outcome. Ann Surg. 2010;251(3):454-60. https://doi.org/10.1097/SLA.0b013e3181c79403.

27. Nakano H, Oussoultzoglou E, Rosso E, et al. Sinusoidal injury increases morbidity after major hepatectomy in patients with colorectal liver metastases receiving preoperative chemotherapy. Ann Surg. 2008;247(1):118-24. https://doi.org/10.1097/SLA.0b0 13e31815774de.

28. Wicherts DA, de Haas RJ, Sebagh M, et al. Regenerative nodular hyperplasia of the liver related to chemotherapy: impact on outcome of liver surgery for colorectal metastases. Ann Surg Oncol. 2011;18(3):659-69. https://doi.org/10.1245/s10434-010-1385-5.

29. Ferrero A, Viganò L, Polastri R, et al. Postoperative liver dysfunction and future remnant liver: where is the limit? Results of a prospective study. World J Surg. 2007;31(8):1643-51. https://doi. org/10.1007/s00268-007-9123-2.

30. Takamoto T, Hashimoto T, Ichida A, Shimada K, Maruyama Y, Makuuchi M. Surgical strategy based on indocyanine green test for chemotherapy-associated liver injury and long-term outcome in colorectal liver metastases. $J$ Gastrointest Surg. 2018;22(6):1077-88. https://doi.org/10.1007/s11605-018-3712-2.

31. Leung U, Simpson AL, Araujo RL, et al. Remnant growth rate after portal vein embolization is a good early predictor of posthepatectomy liver failure. J Am Coll Surg. 2014;219(4):620-30. h ttps://doi.org/10.1016/j.jamcollsurg.2014.04.022.

32. Simpson AL, Adams LB, Allen PJ, et al. Texture analysis of preoperative CT images for prediction of postoperative hepatic insufficiency: a preliminary study. $J$ Am Coll Surg. 2015;220(3):339-46. https://doi.org/10.1016/j.jamcollsurg.2014. 11.027.

33. Zhu WS, Shi SY, Yang ZH, et al. Radiomics model based on preoperative gadoxetic acid-enhanced MRI for predicting liver failure. World J Gastroenterol. 2020;26(11):1208-20. https://doi. org/10.3748/wjg.v26.i11.1208.

Publisher's Note Springer Nature remains neutral with regard to jurisdictional claims in published maps and institutional affiliations. 